\title{
Qtc Changes in Smokers and Non Smokers - A Comparativestudy
}

\author{
${ }^{1}$ Arvind Thangarasa, ${ }^{2}$ Renuka Devi.M.R, ${ }^{3}$ P.Sai Kumar \\ ${ }_{1,2,3,4}$ Sree Balaji Medical College and Hospital,Bharath University, Chennai,India
}

\begin{abstract}
We did a study comparing QTC in smokers and non smokers. We included 88 Healthy male volunteers-44 smokers and 44 non smokers of age from 18 to 30 years from the opd of Sree Balaji Medical College \& Hospital. Healthy male volunteers including smokers as per ICD - 10 criteria for substance abuse were included.Subjects with systemic illness (DM, HTN,TB, BA, CAD etc), drug and alcohol intake were excluded. With the subjects in resting supine position a 12 lead Electrocardiogram using single channel ECG cardiant(heartview 1200 ECG recorder-manufactured by browndove healthcare pvt.Ltd) was recorded in the Research Lab of SBMC\&H. QTC was compared between smokers and non smokers. There is no significant change in QTc of smokers when compared to non smokers in our study.
\end{abstract}

Keyword: Electrocardiogram, QTc, Smokers

\section{Introduction}

The World Health Organization predicts that tobacco deaths in India may exceed 1.5 million annually by 2020 . .63\% of all deaths are caused by NCDs, for which tobacco use is one of the greatest risk factors(4). Greater than 600000 people die each year from exposure to second-hand smoke. The are more than 120 million smokers in India, the rate of tobacco-associated deaths in India is increasing at about 3\% per year(6) .

\section{Background}

Electrocardiogram (ECG) is one of the easiest and non invasive investigations to perform. Various studies have already been done looking for changes in ECG of smokers compared to non smokers. All these studies show varying changes and it is necessary to establish changes in ECG of smokers. A similar study(2) was conducted in General population of Davangere in 2010 which shows some changes in ECG of smokers. Another study (1) done in Dhaka in 2011 concluded that there is no significant changes in ECG of smokers when compared to that of non smokers. With this background we did a study comparing only QTc in smokers and non smokers

AIM

To study QTc changes in smokers compared to non smokers.

\section{Objective}

To make ECG recording in smokers

To make ECG recording in non smokers

To compare QTc between smokers and non smokers

\section{Materials}

We included 88 Healthy male volunteers- 44 smokers and 44 non smokers of age - from 18 to 30 years from the opd of Sree Balaji Medical College \& Hospital . Healthy male volunteers including smokers as per ICD - 10 criteria for substance abuse were included.Subjects with systemic illness(DM,HTN,TB,BA,CAD etc),drug and alcohol intake were excluded. With the subjects in resting supine position a 12 lead Electrocardiogram using single channel ECG cardiant(heartview 1200 ECG recorder-manufactured by browndove healthcare pvt.Ltd) was recorded in the Research Lab of SBMC\&H. QTc was compared between smokers and non smokers

\section{Methods}

Instituitional Ethical committee approval was obtained. Written Informed consent was obtained from subjects. A thorough clinical and systemic examination was done. All subjects were asked to abstain from smoking and caffeine beverages $2 \mathrm{hrs}$ prior to ECG recording. With the subjects in resting supine position a 12 lead Electrocardiogram using single channel ECG cardiant(HeartView 1200 ECG recorder-manufactured by browndove healthcare pvt.Ltd) was recorded in the Research Lab of SBMC\&H. QTc was calculated using Bazett`s formula. QTc was compared between smokers and non smokers. 


\begin{tabular}{|c|c|c|c|}
\hline \multicolumn{4}{|c|}{ III. Results: } \\
\hline PARAMETERS & SMOKERS & NON SMOKERS & P VALUE \\
\hline QTe & $0.42 \pm 0.03$ & $0.43 \pm 0.02$ & 0.121 \\
\hline QT & $0.37 \pm 0.02$ & $0.34 \pm$ & 0.000* \\
\hline
\end{tabular}

\section{Discussion}

Venkatesh et al (2)showed there is no significant change in QTc Interval of smokers compared to non smokers.Our study also has the same finding.Khan IS et al(1) did a similar study which showed no change in ECG of smokers compared to non smokers.our study has some significant change in QT but not in QTc. Karjalainen $\mathrm{J}$ et al(5) did a study and concluded prolonged QT as a risk factor for cardiac mortality in smokers.Our study is in acceptance with this .

\section{Conclusion}

There is no significant change in QTc of smokers when compared to non smokers in our study.

\section{References}

[1] Study of ECG Changes in Apparently Healthy Adult Male Smokers*Khan IS,1 Rahman MA,2 and Amin R

[2] A Study of Electrocardiographic changes in smokers compared to normal human beings. Venkatesh $\mathrm{G}^{*}$ and Swamy RM** Biomedcal Research 2010; 21 (4): 389-392

[3] Diagnostic criteria for research. ICD-10 Classification of mental and behavioral disorders WHO Geneva $1993: 75$.

[4] World Health Organization. Geneva: World Health Or-ganization; 2008. WHO report on the global tobacco epidemic?

[5] QT interval as a cardiac risk factor in a middle aged population.Karjalainen J, Reunanen A, Ristola P, Viitasalo M .Heart.1997.. Jun;77(6):543-8

[6] By Peggy Peck, Executive Editor, MedPage TodayPublished: February 14, 2008 